Dhaka Univ. J. Sci. 61(1): 35-40, 2013 (January)

\title{
Chemotherapy Drug Scheduling: A Particle Swarm Optimization Approach
}

\author{
M.S. Alam ${ }^{1}$, S. Algoul ${ }^{2}$, M.A. Hossain ${ }^{3}$ and M.A.A. Majumder ${ }^{4}$ \\ ${ }^{1}$ Department of Applied Physics, Electronics and Communication Engineering, University of Dhaka, \\ Dhaka-1000, Bangladesh \\ ${ }^{2}$ Department of Computing, University of Bradford, UK, ${ }^{3}$ Department of Computer Science, University of Northumbria, UK, \\ ${ }^{4}$ Department of Life Science, University of Bradford, UK, \\ msalam@univdhaka.edu
}

Received on 29. 09. 2011. Accepted for Publication on 10. 07. 2012

\begin{abstract}
This paper presents an investigation into a model of chemotherapy drug scheduling for cancer treatment using feedback control and a bioinspired optimization algorithm, called Particle Swarm Optimization (PSO). The main aim of chemotherapy treatment is to reduce the tumour size. Earlier a feedback control method, namely Integral-Proportional-Derivative (I-PD) was developed in [1] for non-phase specific tumour model to control the drug to be infused into the patient's body by maintaining a tolerable level of drug concentration at tumour sites. In this work, PSO is used to devise an effective drug scheduling for a phase-specific tumour model by optimizing the parameters of earlier developed I-PD controller. The phase specific cancer tumour model is used to analyze the effects of drug on different cell populations, plasma drug concentration and toxic side effects. Results show that proposed method can reduce the tumour size up to $72 \%$ with relatively lower drug doses. Moreover, the method results very low toxic side effects throughout the whole period of treatment.
\end{abstract}

Keywords: Cancer chemotherapy, Cell compartment, Drug scheduling, Feedback control, Particle Swarm optimization.

\section{Introcuction}

Chemotherapy is one of the essential treatment methods for cancer. Traditionally one or more drugs are infused to the body. The efficiency of the dosages of the treatment is often measured as the interval of time from the start of therapy, until the end of treatment. A number of models have been developed and used to characterise the evolution and effects of treatment on cancer by dividing the tumour into number of compartments (phase-specific) as reported in $[2,3]$. The cell compartment models of cancer cells population show transition rates between proliferating and quiescent cells as non-specified functions of the total population. In [4,5], Dua and coworkers have presented phase-specific treatment models for cancer chemotherapy. The model considers the cell cycle which consists of more than one compartment to take into account the type of cells that are affected by the drug.

The main aim of chemotherapy treatment is to eradicate or minimize the number of cancer cells after a number of fixed treatment cycles. Conventional clinical methods of chemotherapy can hardly find optimum doses that can minimize cancerous cells and toxic effects simultaneously. Researchers have employed/used genetic algorithm (GA) in scheduling chemotherapy drug doses and results are reported in [1,6-8]. The drawbacks of GA, such as, complexity in algorithm, huge computational resources, long execution time, premature convergence to local minima due to lack of diversity in population etc, have led the current researchers in search for more efficient and effective algorithms and techniques.

Particle swarm optimization (PSO) [11], one of the relatively new evolutionary computation techniques, has been successfully used as an optimisation and design technique in various fields [11], mainly due to its simplicity, low computational cost, fast convergence and good overall performance [12]. These features motivated the current researchers to explore the potential of PSO in chemotherapy drug scheduling.

This paper investigates the method of chemotherapy drug scheduling for cancer treatment using feedback control and PSO. A variant of Proportional-Integral-Derivative (PID), namely Integral-Proportional-Derivative (I-PD)[1] has been used to control the drug to be infused to the patient's body. A phase specific cancer tumour model, commonly known as compartmental model [4] is used for this work. The parameters of the I-PD controller are optimized by PSO to improve the tumour response as well as other treatment objectives.

\section{Mathematical Model}

The cell cycle is a chain of phases that both normal and cancer cells undergo from their birth to death $[2,4]$ as show in Figure 1. The first stage refers to the gap G1, in which stage the cell prepares for DNA synthesis. In the second stage called $\mathrm{S}$ the DNA synthesis takes place in preparation for cell division (many anticancer drugs act by interfering with DNA at this stage, causing cell death). The third stage, gap G2, is specialized to prepare the proteins and RNA for cell division. In the fourth stage $M$, the cell division takes place to produce two identical daughter cells. The last stage called Go is a resting phase in which cell is quiescent, viable but unable to divide. The tissue, in general, contains three different types of cells: the proliferating cells, the quiescent cells and the dead cells. Figure 2 shows a two-compartment model where $\mathrm{P}$ (Proliferating) represents the combination of the first four stages of the cell cycle as mentioned earlier $\left(\mathrm{G}_{1}, \mathrm{~S}, \mathrm{G}_{2}\right.$ and $\left.\mathrm{M}\right)$ and Q (Quiescent cells) indicates stage $\mathrm{G}_{0}$. The proliferating part contains actively dividing cells whereas quiescent part are inactive cells (Go), but capable of dividing if a certain stimulus is given. The dead cells are unable to divide because they have completed their life cycle. The parameters $m$ and $b$ express the immigrants between the proliferating cells and quiescent cells respectively. Here ' $a$ ' indicates the growth rate of cycling cells and $n$ is the natural decay of the cycling cells. A number of differential equations used to build a two 
compartment model of cancer chemotherapy treatment are explained briefly. The first equation, predicts the rate of change of proliferation cells population at the tumour site during the treatment, is as follows [4]:

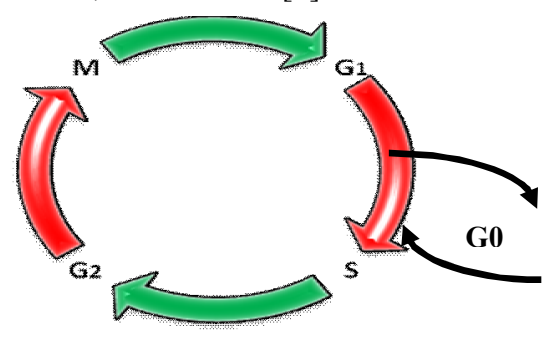

Fig. 1. Different phases of cell cycle

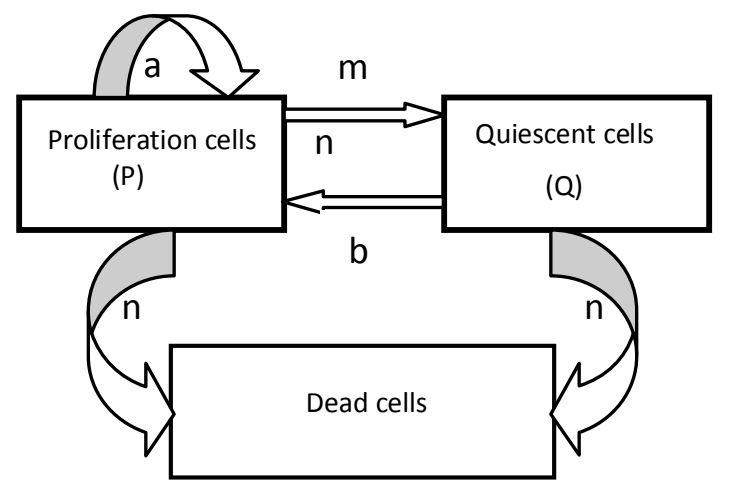

Fig. 2. Two compartment cancer cells model

$$
\begin{aligned}
& \frac{d P}{d l}=(a-m-n) P(t)+b Q(t)-g(t) P(t) \\
& P(0)=P_{0}
\end{aligned}
$$

Here $\mathrm{P}$ and $\mathrm{Q}$ represent population of proliferating and quiescent cells whereas $\mathrm{g}(\mathrm{t})$ represents cell killing rate. The rate of change of cell population in the quiescent compartment is represented by:

$$
\frac{\mathrm{dQ}}{\mathrm{dl}}=\mathrm{mP}(\mathrm{t})-\mathrm{bQ}(\mathrm{t}), \quad \mathrm{Q}(0)=Q_{0}
$$

A logistic equation is used to describe the effect of drug on normal cells, as expressed by equation (3) below:

$$
\begin{gathered}
\frac{d Y}{d t}=\delta Y(t)\left(1-\frac{Y(t)}{K}\right)-g(t) Y(t) \\
Y(0)=Y_{0}
\end{gathered}
$$

Here $Y(t)$ indicates the normal cells population whereas $\delta$ and $K$ represent the growth rate and the carrying capacity of normal cells respectively. $Y(0)$ is the initial value of normal cell population at the beginning of the treatment. Equation (4) shows the rate of change of drug concentration at the tumour site during the treatment cycle.

$$
\frac{d D}{d t}=u(t)-\gamma D(t)
$$

where $u(t)$ is the amount of drug doses to be infused to patient's body and $\gamma$ is drug decay which is related to the metabolism of drug inside patient's body. Drug concentration at the instant before chemotherapy begins is, $D(0)=D_{0}$, at $t=$ 0 , It is noted that $D_{0}$ is assumed to be zero as suggested by Martin and Teo in [2]. The relationship between cell killing rate and drug concentration at the tumour site is [2]:

$g(t)=k_{1} D(t)$

where $k_{1}$ is a constant related to effect of drug concentration on cell killing. Toxicity, $\mathrm{T}(\mathrm{t})$, developed during the chemotherapy treatment is quantitatively modelled from drug concentration as [2].

$$
\frac{d T}{d t}=D(t)-\eta T(t)
$$

where $T(t)$ is the level of toxicity developed inside the patient's body due to chemotherapy drug and parameter $\eta$ indicates the rate of elimination of toxicity.

To make the treatment effective and limit the adverse side effects, several constraints are imposed on drug concentration and toxicity to the model.

These constraints are as follows:

$$
\begin{aligned}
& D(t) \leq 50 \\
& T(t) \leq 100
\end{aligned}
$$

It is to be noted that, for phase-specific chemotherapy treatment, these constraints have been derived from clinical data by Dua in [5] and Tse and co-workers [6] and used/cited by many researchers in similar research $[1,2,4,8]$. The limiting value of normal cell which should be maintained throughout the period of treatment is represented by

$$
\mathrm{Y}_{\min } \leq \mathrm{Y}(\mathrm{t}) \leq \mathrm{K} \text {, for all } \mathrm{t} \in[0, \mathrm{~T}]
$$

The parameter $Y_{m \text { mn }}$ indicates the minimum number of the normal cells at tumour site. Using above equations, a Matlab/Simulink model [13] was developed with parameters and values as illustrated in Table I.

\section{Control Scheme}

A schematic diagram of chemotherapy drug scheduling scheme is shown in Figure 3. An I-PD controller involves three parameters, the proportional gain $k_{p}$, integral gain $k_{i}$, and derivative gain $k_{\alpha}$, and is designed to maintain a predefined level of drug concentration at tumour sites.

Drug concentration at the tumour is used as the feedback signal to the controller which is compared with a predefined reference level. The difference between these two is called the error which is used as input to the controller. The output of the controller $u(t)$ is formed by taking three terms as: 


$$
\mathrm{u}(\mathrm{t})-\mathrm{K}_{\mathrm{t}} \int_{0}^{\mathrm{t}} \rho(\mathrm{t}) \mathrm{dt}-\left[\mathrm{K}_{\mathrm{d}} \frac{\mathrm{d}}{\mathrm{dt}} \mathrm{D}(\mathrm{t})+\mathrm{K}_{\mathrm{p}} \mathrm{D}(\mathrm{t})\right]
$$

Where $e(t)$ is the error which is the difference between reference $X_{D}$ and drug concentration $D(t)$ as:

$$
e(t)=\left(X_{D}-D(t)\right)
$$

It is noted that $X_{D}$ indicates reference signal to the controller which can be depicted as the desired drug concentration to be maintained at the tumour site during the whole period of treatment.

Table. 1. Parameters of phase-specific cancer tumour model [4]

\begin{tabular}{|l|l|r|}
\hline \multicolumn{2}{|l|}{ Parameters } & Values \\
\hline $\mathrm{a}$ & growth rate of Proliferating cells & $0.5 \mathrm{day}^{-1}$ \\
\hline $\mathrm{m}$ & $\begin{array}{l}\text { mutation rate of proliferating } \\
\text { cells to quiescent cells }\end{array}$ & $0.218 \mathrm{day}^{-1}$ \\
\hline $\mathrm{n}$ & natural end of the cycling cells & $0.477 \mathrm{day}^{-1}$ \\
\hline $\mathrm{b}$ & $\begin{array}{l}\text { mutation rate of quiescent cells } \\
\text { to proliferating cells }\end{array}$ & $0.05 \mathrm{day}^{-1}$ \\
\hline$\delta$ & growth rate of normal cell & $0.1 \mathrm{day}^{-1}$ \\
\hline$\lambda$ & growth rate of cancer cells & $0.8 \mathrm{day}^{-1}$ \\
\hline $\mathrm{K}$ & carrying capacity of normal cell & $10^{9}$ \\
\hline $\mathrm{P}$ & proliferating cells population & $0.2 \times 10^{12}$ \\
\hline $\mathrm{Q}$ & quiescent cells population & $0.8 \times 10^{12}$ \\
\hline $\mathrm{Y}$ & normal cells population & $10^{9}$ \\
\hline$Y_{\text {min }}$ & limitation of normal cells & $10^{8}$ \\
\hline
\end{tabular}

The reference to the controller is chosen in such a way as to limit the drug concentration in the plasmas indicated in equation (7) which in turn limits the toxic side effects. Using equations (4), (5) and (9) in equation (1) and solving for $P(t)$ gives:

$P(t)=(a-m-n) P(t)+b Q(t)$

$-K_{1}\left(K_{i}\left(\int_{0}^{t} e(t) d t-\left[K_{d} \frac{d}{d t} X_{2}(t)+\right]\right)-\gamma V_{1}(t)\right) P(t)$

Equation (11) shows the interaction between the parameters of I-PD controller and the cells reduction. The third term of (11) expresses how the parameters of the controller affect the rate of cells reduction.

The output of the I-PD control, which is chemotherapy drug dose, is applied to the model to observe its effects. It is required to tune three parameters $k_{i}, k_{p}$ and $k_{i}$ of IPD controller to achieve desired performance. In this work, PSO is used to optimize parameters of I-PD controller that in turn improves the drug scheduling as well as treatment. It is important to note that the whole control scheme and drug scheduling is designed for a period of 84 days as recommended by many researchers $[1,2,6]$.

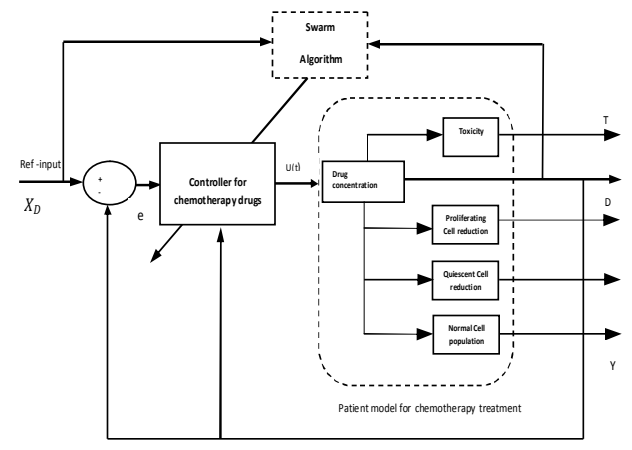

Fig. 3. Schematic diagram of drug scheduling scheme for cancer treatment

\section{Particle Swarm Optimization}

The PSO algorithm works on the social behaviour of particles in the swarm. The position vector and the velocity vector of the $i$-th particle in the $d$-dimensional search space can be represented as $X_{i}=\left(x_{i 1}, x_{i 2}, \ldots \ldots \ldots x_{i i}\right) \quad$ and $V_{i}=\left(v_{i \mathrm{~L}}, v_{i 2}, \ldots \ldots v_{i d}\right)$ respectively. According to a user defined fitness function, let the best position of each particle be, $P_{i}=\left(p_{i 1}, p_{i 2}, \ldots \ldots \ldots p_{i d}\right)$ and the fittest particle found so far at time $t$ be $P_{g}=\left(p_{g 1}, p_{g 2}, \ldots \ldots \ldots p_{g d}\right)$. Then, the new velocities and positions of the particles for the next fitness evaluation are calculated using the following two equations [9, 10]:

$v_{i d}=v_{i d}+C_{1} \times r 1 \times\left(P_{i d}-x_{i d}\right)$

$+C_{2} \times r 2 \times\left(P_{g e}-x_{i d}\right)$

$x_{i d}=x_{i d}+v_{i d}$

where $C_{1}$ and $C_{2}$ are constants known as acceleration coefficients, and $r 1$ and $r 2$ are two separately generated uniformly distributed random numbers in the range $(0,1)$. Initially, a population of particles is generated with random positions, and then random velocities are assigned to each particle. The fitness of each particle is then evaluated according to a user defined objective function. At each generation, the velocity of each particle is calculated according to equation (12) and the position for the next function evaluation is updated according to equation (13). Each time if a particle finds a better position than the previously found best position, its location is stored in the memory. Generally, a maximum velocity $v_{\operatorname{maxd}}$ for each modulus of the velocity vector of the particles $v_{i d}$ is defined in order to control excessive roaming of particles outside the user defined search space. Whenever a $v_{i d}$ exceeds the defined limit, its velocity is set to $v_{\text {maxd }}$. In order to control the global search and convergence to the global best solution, a derivative of particle swarm algorithm that uses time-varying inertia weight factor $w$ and time-varying acceleration coefficients $C_{1}$ and $C_{2}$ [14] is used in this work. The mathematical representation of this modified PSO is given as:

$v_{i d}=\omega \times v_{i d}+C_{1} \times r 1 \times\left(P_{i d}-x_{i d}\right)$ 


$$
\begin{aligned}
& +c_{2} \times r 2 \times\left(P_{g d}-x_{i d}\right) \\
& w=w_{2}+\left(w_{1}-w_{2}\right) *(\text { MAXTTER-iter }) / \text { MAXTTER } \\
& c_{1}=c_{1 i}+\left(c_{1 f}-c_{1 i}\right) * \text { iter } / \text { MAXTTER } \\
& c_{2}=c_{2 i}+\left(c_{2 f}-c_{2 i}\right) * i t e r / M A X T T E R
\end{aligned}
$$

where $w_{1}$ and $w_{2}$ are the initial and final values of the inertia weight, respectively, $c_{1 i}, c_{1 f}, c_{2 i}$, and $c_{2 f}$ are constants, itor is the current iteration number and MAXTTER is the maximum number of allowable iterations. In [14], it has been demonstrated that the optimal solution can be improved by varying the value of $w$ from 0.9 at the beginning of the search to 0.4 at the end of the search for most problems. An improved optimum solution for most of the benchmarks was observed when changing $\sigma_{1}$ from 2.5 to 0.5 and changing $c_{2}$ from 0.5 to 2.5 , over the full range of the search [14]. The commonly used PSOs are either global version or local version of PSO. In this work, the ring topology is used to find the best guide for any particle while updating its velocity. Details of the algorithm can be found in [16].

\section{Implementation}

An initial swarm of dimension $20 \times 3$ is created where number of particles and parameters in each individual are 20 and 3 respectively. Each parameter is randomly generated within a range of $(0,2)$. Each particle represents a solution where three elements are assigned to proportional gain $k_{p}$, second to integral gain $k_{i}$, and third to derivative gain $k_{d}$ respectively as indicated in equation (9). The error $e(t)$ as indicated in equation (10) is used to form the objective function $f(x)$ of the optimization process. Mean squared error (MSE) is used as the objective function. This is given as:

$$
\operatorname{MSE} f(x)=\frac{1}{x} \sum_{i=t}^{N}(\mathrm{~g}(\mathrm{t}))^{2}
$$

Toxicity is assumed to be inversely proportional to the number of normal cells. For compartment models, the normal cell population is required to be maintained equal or more than $10^{8}$ during the whole treatment cycle $(84$ days)[6]. This can be termed as threshold value which is set as a constraint in the PSO optimization process along with constraints indicated in equation (7). To extract acceptable solution, prior to calculating fitness function, the normal cell population of each individual is checked and if it is less than the threshold value then that particle (solution) is penalized.

Matlab coding [13] have been used to implement the proposed scheme and the optimization process. The maximum number of generations was set to 300 . PSO optimization process with abovementioned parameters is run five times on the same model to show its effectiveness and repeatability in such problem. The best objective function of each run is recorded at the end of maximum generation and shown in Table-II. To assess the reproducibility of the PSO optimization process, standard deviation of best objective functions of all runs is calculated which is 0.567 . It is noted that the standard deviation is low compared to values of best objective function which exhibits the reproducibility of the PSO optimization process. Figure 4 shows algorithm convergence in different runs and Table II shows the results for different runs.

The average level of drug dosages infused to the patient's body is nearly 4.27 while the maximum dose is 5.52 as recorded in run-3. The average level of drug concentration for all runs is nearly 11.54 which are effective but much lower than the limiting value. It is observed that the percentage of proliferating cells reduction is $72 \%$ for most of the runs with run-5 records maximum reduction of $72.1 \%$. The percentage of quiescent cells reduction is slightly lower, $60 \%$ for most of the runs with run-3 yields minimum reduction of $53 \%$. The average level of toxicity for different runs is nearly 32 whereas maximum level of

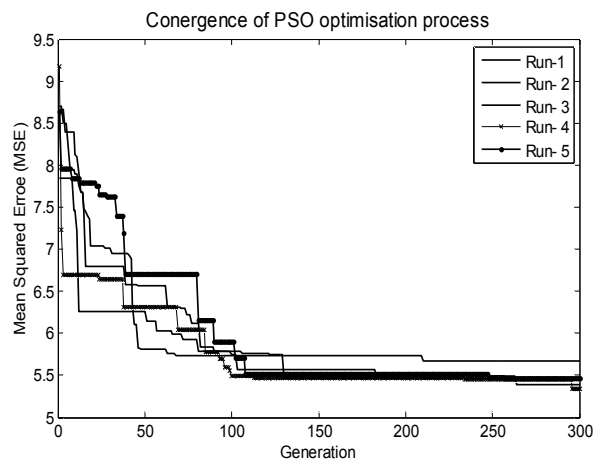

Fig. 4. Convergence of PSO optimization process

toxicity varies within a range of 64.95 to 68.07 . It is mentioned that the minimum level of toxicity was recorded in run-3 whereas maximum level of toxicity was observed in run 4. The number of normal cells is more than the threshold value $\left(=10^{8}\right)$ in all runs. It is important to observe that, drug scheduling with run-5 reduces both the proliferating and quiescent cells to the minimum level whereas the level of toxicity and drug concentrations are relatively lower.

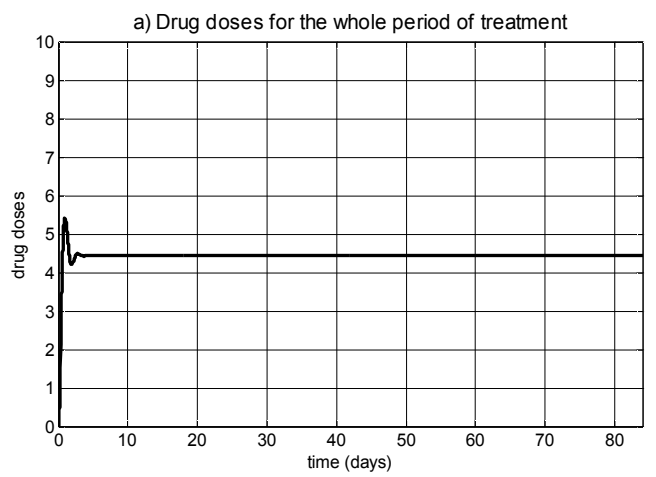

(a) Drug dosages for the whole period of treatment. 


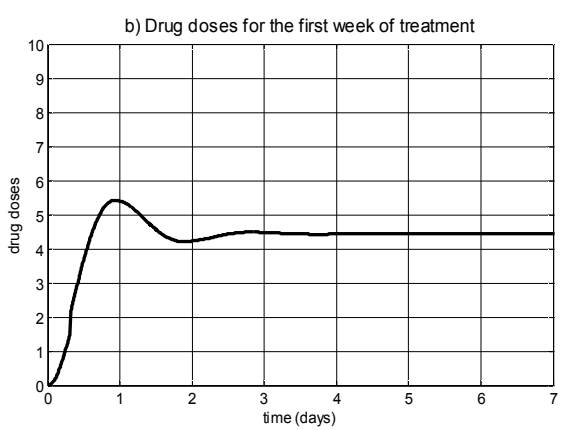

(b) Drug doses for first week of treatment.

Fig. 5. Drug doses

\section{Results}

The drug dosages obtained with the controller parameters at the end of run-5 is applied to the compartment model and response is observed. The drug scheduling throughout the treatment period is shown Figure 5(a). For more clarity, the drug dosage for the first week of treatment is shown in figure $5(\mathrm{~b})$. It is observed that the drug dosage is initially zero and gradually increases with time and records a highest value on day- 1 , which is $5.45 \mathrm{D}$. The drug dosage slightly decreases on day- 2 and then becomes almost stable for remaining period. The average value of drug dosages is recorded as 4.27D and is shown in Table II.

The effects of the drug concentration at the tumour site throughout the treatment period are shown in Figure 6. The drug concentration at the tumour site is initially zero and record maximum value of 14.74 on day-1. It is noted that although the drug concentration exceeds the set point (on day-2), it is still much lower that the limiting value as indicated in equation (5).

The maximum level of toxicity throughout the treatment period was recorded as 65.50 at the first day of treatment while the average value for the whole period is 32.97 . The level of toxicity for the whole period of treatment cycle is shown in Figure 7. It may be noted that both the maximum and average values are too low to cause any harmful toxic side effects. The reductions of proliferating cells, quiescent cells and normal cells are shown in Figures 8(a), (b) and (c) respectively. It may be noted that the reductions are also different, with proliferating cells showing maximum reduction followed by quiescent cells.

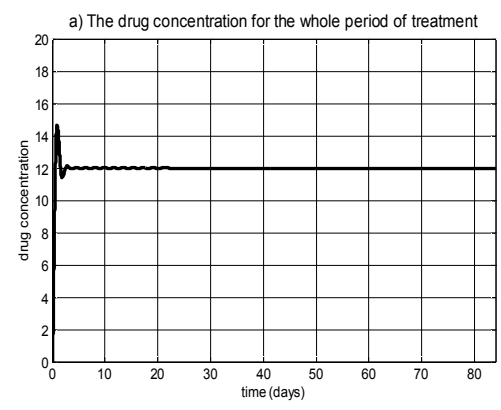

Fig. 6. Drug concentration

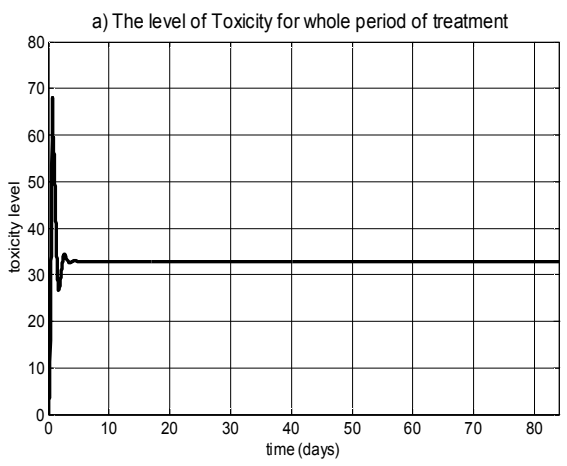

Fig. 7. The toxicity level during treatment

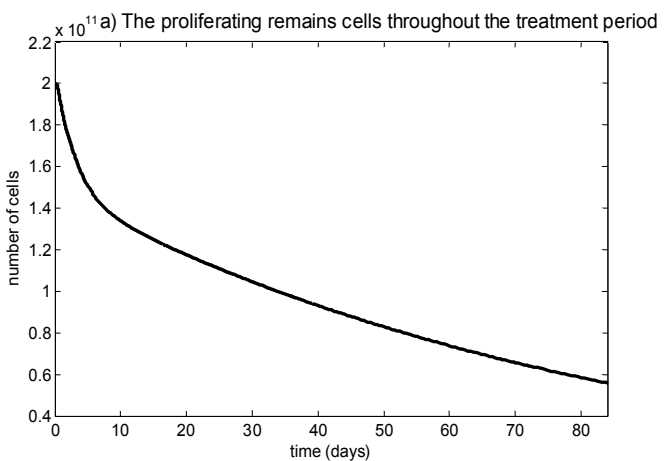

(a) Reduction of proliferation cell

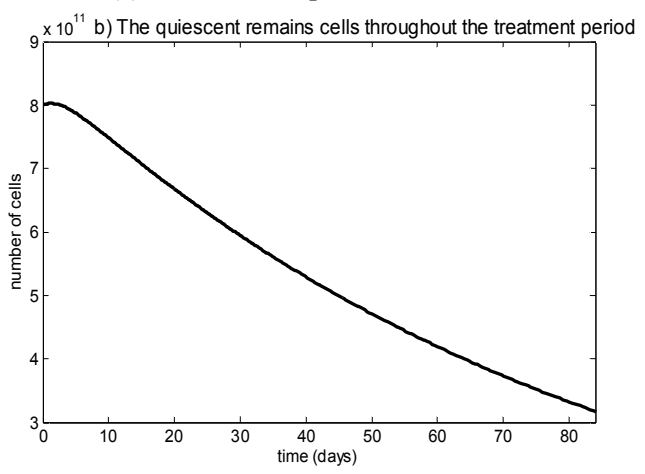

(b) Reduction of quiescent cell

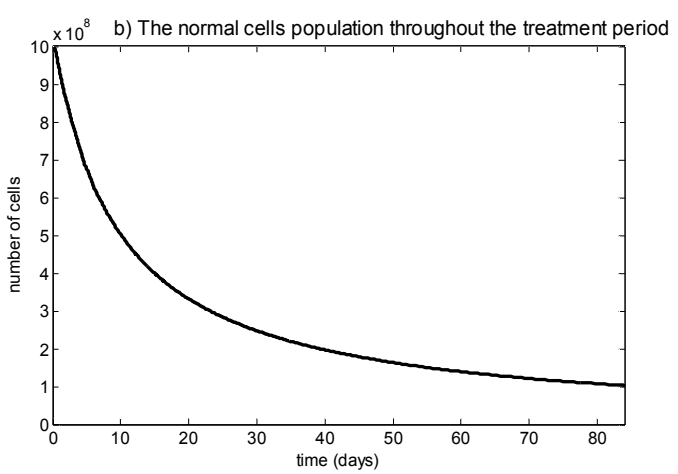

(c) Reduction of normal cells

Fig. 8. Effects of chemotherapy drug scheduling on different types of cells 
Table. 2 Simulation results of different runs

\begin{tabular}{|l|l|l|l|l|l|l|l|l|l|l||}
\hline \hline $\begin{array}{l}\text { No. } \\
\text { of } \\
\text { runs }\end{array}$ & \multirow{2}{*}{$\begin{array}{l}\text { Best } \\
\text { Obj }\end{array}$} & \multicolumn{2}{l|}{ drug doses } & \multicolumn{2}{l|}{$\begin{array}{l}\text { drug } \\
\text { concentration }\end{array}$} & \multicolumn{2}{l|}{ Toxicity } & $\begin{array}{l}\text { Reduction } \\
\text { of } \\
\text { Proliferation } \\
\text { cells }\end{array}$ & $\begin{array}{l}\text { Reduction of } \\
\text { Quiescent } \\
\text { cells }\end{array}$ & $\begin{array}{l}\text { Normal } \\
\text { cells }\end{array}$ \\
\cline { 3 - 12 } & Max & Avg & Max & Avg & Max & Avg & & \\
\hline Run1 & 5.46 & 5.30 & 4.25 & 14.35 & 11.54 & 66.34 & 32.90 & $72.1 \%$ & $60.3 \%$ & $1.027 \times 10^{8}$ \\
\hline Run2 & 5.38 & 5.26 & 4.25 & 14.23 & 11.54 & 67.56 & 32.90 & $72.1 \%$ & $60.3 \%$ & $1.027 \times 10^{8}$ \\
\hline Run3 & 5.38 & 5.52 & 4.26 & 14.94 & 11.55 & 64.95 & 32.95 & $67.2 \%$ & $53.3 \%$ & $1.214 \times 10^{8}$ \\
\hline Run4 & 5.33 & 5.41 & 4.27 & 14.64 & 11.57 & 68.07 & 33.01 & $70.5 \%$ & 58.1 & $1.086 \times 10^{8}$ \\
\hline Run5 & 5.46 & 5.45 & 4.27 & 14.74 & 11.57 & 65.50 & 32.97 & $72.1 \%$ & $60.3 \%$ & $1.027 \times 10^{8}$ \\
\hline
\end{tabular}

\section{Conclusions}

This paper has presented an investigation into the development of a feedback I-PD controller for chemotherapy drug scheduling where PSO has been used to optimize the parameters of the controller. Model based on the cells function has been used to analyses the effects of the drug scheduling designed by the controller. It is noted that the obtained drug schedule is continuous in nature having lower and nearly stable value throughout the whole period of treatment. The proposed drug scheduling pattern has reduced the number of tumour cells significantly with the tolerable drug concentration and toxicity level. Finally, similar method/strategy can be extended for multidrug or combination chemotherapy regimen. Future work will include verification of the proposed scheduling with clinical data and experiments and efforts are underway in that direction.

1. Algoul, S., M.S. Alam, M.A. Hossain and M.A. Majumder, 2010, Multi-objective Optimal Chemotherapy Control Model for Cancer Treatment, Springer Journal on Medical and Engineering and Computing; 49(1), 51-65, Springer Verlag, Berlin.

2. Martin, R. and K. Teo, 1994, Optimal control of drug administration in chemotherapy tumour Growth, First ed.,World Scientific, 95-111.

3. Panetta, J., 1999, A mathematical model of drug resistance: Heterogeneous tumour, Math. Biosci 147, 41-61.

4. Dua, P., V. Dua and N. Pistikopoulos, 2008, Optimal delivery of chemotherapeutic agents in cancer, Computer and Chemical Engineering, 32, 99-107.

5. Dua, P., 2005, Model based and parametric control for drug delivery systems, $\mathrm{PhD}$ Thesis, Imperial College London, UK.
6. Tes, S., Y. Liang, K.-S. Leung, K. Lee, and T. S. K. Mok, 2007, A Memetic Algorithm for Multiple-Drug Cancer Chemotherapy Scheduling

7. Optimization, IEEE Trans on Systems, Man, and Cybernetics-B, 37, 84-91.

8. McCall, J., A. Petrovski and A. Shakya, 2008, Evolutionary Algorithms for Cancer Chemotherapy Optimization, Computational Intelligence in Bioinformatics, 265-296.

9. Algoul, S., M.S. Alam, K. Sakib, K., M.A. Hossain, and M.A. Majumder, 2011, MOGA-based Multi-drug Optimisation for Cancer Chemotherapy, Advances in Intelligent and Soft Computing, 93, 133-140, Springer Verlag, Heidelberg.

10. Kennedy, J. and R. Eberhart, 1995, Particle swarm optimization, Proceedings of IEEE International Conference on Neural Networks, 1942-1948, IEEE Press, Piscataway, NJ.

11. Kennedy, J. and R. Eberhart, 2001, Swarm Intelligence, Morgan Kaufmann Publishers.

12. Lazinica, A., 2009, Particle Swarm Optimization, (Edited), Published by In-Tech, Croatia, January.

13. Shi, Y. and R. Eberhart, 1998, A modified particle swarm optimizer, Proc. IEEE Int. Conf. Evolutionary Computation, 69-73.

14. The Mathworks, Inc., 2010 "MATLAB Reference Guide."

15. Ratnaweera, A., K. Halgamuge, and C. Watson, 2004, Self-organizing hierarchical particle swarm optimizer with time varying accelerating Coefficients, IEEE Transactions on Evolutionary Computation, 8(3), 240-255.

16. Alam, M.S. and M.O. Tokhi, 2007, Modelling of a twin rotor system: a particle swarm optimisation approach, Proceedings of the Institution of Mechanical Engineers, Part G: Journal of Aerospace Engineering, 221(3), 353374. 
Chemotherapy Drug Scheduling: A Particle Swarm Optimization Approach 